PROCEEDINGS OF THE

AMERICAN MATHEMATICAL SOCIETY

Volume 126, Number 4, April 1998, Pages 1219-1230

S 0002-9939(98)04174-4

\title{
CODING NESTED MIXING ONE-SIDED SUBSHIFTS OF FINITE TYPE AS MARKOV SHIFTS HAVING EXACTLY THE SAME ALPHABET
}

\author{
ALEJANDRO MAASS AND SERVET MARTÍNEZ \\ (Communicated by Mary Rees)
}

\begin{abstract}
Let $X_{0}, X$ be mixing one-sided subshifts of finite type such that $X_{0} \subseteq X$. We show a necessary and sufficient condition for the existence of mixing Markov shifts $Y_{0}, Y, Y_{0} \subseteq Y$, and a conjugacy $\pi: Y \rightarrow X$ with $\pi\left(Y_{0}\right)=X_{0}$, such that the sets of letters appearing in both systems are the same, more precisely, $L_{1}\left(Y_{0}\right)=L_{1}(Y)$.
\end{abstract}

\section{INTRODUCTION}

In this paper we address a question of codification of subshifts of finite type. Given two nested mixing one-sided subshifts of finite type, $X_{0} \subseteq X$, we study the conditions for the existence of mixing Markov shifts $Y_{0}$ and $Y, Y_{0} \subseteq Y$, conjugate to $X_{0}$ and $X$ respectively, with the additional condition of using the same letters. This question is natural when you try to hide one subshift of finite type inside another in some maximal way.

Let us comment on two aspects of this question. First, the recoding of subshifts of finite type as Markov shifts can be achieved by classical methods in Symbolic Dynamics (for both one-sided and two-sided subshifts), in particular, the higher block presentation of subshifts. On the other hand, the restrictions imposed on the languages of the Markov shifts obtained after conjugacy lead us to think of methods of embedding (various theorems are well known in the two-sided case, see [LM]) over some full shifts requiring some orbits of $X_{0}$ to be mapped into periodic points using the whole alphabet. However this process does not take care of the Markov property of the final subshifts obtained. Then it is necessary to develop a mixed method taking care of both conditions.

The article is laid out in three sections. Section 2 is devoted to definitions and a general background in the process of state splitting. The main result is given in section 3 .

\section{Definitions}

In this section we give some tools from Symbolic Dynamics that shall be used in the sequel. We follow closely reference $[\mathrm{LM}]$.

Received by the editors April 16, 1996 and, in revised form, September 23, 1996.

1991 Mathematics Subject Classification. Primary 54H20, 58F03.

(C)1998 American Mathematical Society 
2.1 Subshifts of finite type and conjugacy. Let $A$ be a finite alphabet. We denote by $A^{*}$ the set of finite sequences or words on $A$, and by $|w|$ we mean the length of the word $w \in A^{*}$. A language $L$ is an arbitrary subset of $A^{*}$, and we denote by $L_{n}$ the set of words of length $n$ of $L . A^{\mathbb{N}}$ is the set of infinite sequences $x=\left(x_{i}\right)_{i \in \mathbb{N}}$, where $x_{i} \in A$. This set is endowed with the product topology and the shift action $\sigma: A^{\mathbb{N}} \rightarrow A^{\mathbb{N}}, \sigma(x)=\left(x_{i+1}\right)_{i \in \mathbb{N}}$. For this topology $A^{\mathbb{N}}$ is a compact metric space and $\sigma$ is continuous. The flow $\left(A^{\mathbb{N}}, \sigma\right)$ is called the one-sided full shift. A one-sided subshift is a closed shift-invariant subset $X$ of $A^{\mathbb{N}}$ endowed with $\sigma$. In general, the space $X$ is identified with the dynamical system $(X, \sigma)$. The language associated to the subshift $X$ is $L(X)=\left\{w \in A^{*} \mid \exists x \in X, i \in \mathbb{N}\right.$, such that $\left.x_{i} \ldots x_{i+|w|-1}=w\right\}$. Since we will only consider one-sided subshifts we will omit the adjective "one-sided".

Let $X$ and $Y$ be two subshifts. A factor map from $X$ to $Y$ is a continuous onto map $f: X \rightarrow Y$ such that $f \circ \sigma=\sigma \circ f$. $Y$ is called a factor of $X$. If $f$ is also one-to-one we say that $X$ and $Y$ are conjugate, and $f$ is called a conjugacy. The continuous $\sigma$-commuting maps are characterized as those maps which are defined by a local rule, that is:

$$
\begin{gathered}
\exists r \in \mathbb{N}, \exists f^{*}: L_{r+1}(X) \rightarrow L_{1}(Y), \text { such that } \\
\forall x \in X, \forall i \in \mathbb{N}, f(x)_{i}=f^{*}\left(x_{i} \ldots x_{i+r}\right) .
\end{gathered}
$$

Such a map $f$ is called an $(r+1)$-block map. In what follows we will identify $f^{*}$ with $f$. If $f$ is a conjugacy then $f^{-1}$ is also defined by a local rule.

A subshift $X \subseteq A^{\mathbb{N}}$ is said to be of finite type (SFT) if there exist a positive integer $N$ and a collection $\mathcal{B}$ of words of length $N$, such that $x \in X$ if and only if $x_{i} \ldots x_{i+N-1} \in \mathcal{B}$ for all $i \in \mathbb{N}$. The integer $N$ is called an order of $X$. An SFT of order 2 is called a Markov shift. Every SFT is conjugate with a Markov shift.

A Markov shift (MS) can be defined by a square nonnegative integral matrix $M$, or equivalently by a directed graph $G_{M}$ (this graph has $M_{v v^{\prime}}$ edges from vertex $v$ to vertex $\left.v^{\prime}\right)$. If the set of edges is $E_{M}$ then the MS $X_{M}$ is the set of $x \in\left(E_{M}\right)^{\mathbb{N}}$ such that the terminal vertex of $x_{i}$ is the initial vertex of $x_{i+1}$ for all $i \in \mathbb{N}$. Another way to represent an MS $X$ is by a directed graph where $L_{1}(X)$ is the set of vertices and there is an edge from $a \in L_{1}(X)$ to $b \in L_{1}(X)$ if and only if $a b \in L_{2}(X)$. Then $X$ is the subshift defined by the one-sided infinite walks on the vertices of this graph. It is easy to see that the MS $X_{M}$ defined by the incidence matrix of this graph is conjugate to $X$, and it is called the arc representation of $X$. We recall that the incidence matrix of a graph is the 0-1 matrix indexed by the vertices which has a 1 in the entry corresponding to a pair of vertices if and only if there is an edge between them.

An SFT $X$ is mixing if there is $t_{0} \in \mathbb{N}$ such that for any pair of words $w_{1}, w_{2} \in$ $L(X)$ and any $t \geq t_{0}$ we can find $w \in L(X),|w|=t$, with $w_{1} w w_{2} \in L(X)$. A matrix which defines a mixing MS is irreducible and aperiodic. The mixing SFT using only one letter will be called trivial.

For a subshift $X$ the predecessor and successor sets of $a \in L_{1}(X)$ are $P_{X}(a)=$ $\left\{a^{\prime} \in L_{1}(X) \mid a^{\prime} a \in L_{2}(X)\right\}$ and $S_{X}(a)=\left\{a^{\prime} \in L_{1}(X) \mid a a^{\prime} \in L_{2}(X)\right\}$ respectively. Let us point out that if $X=X_{M}$ then the equality of sets $P_{X}(a)=P_{X}(b)$ holds if and only if the initial vertices of $a$ and $b$ in $G_{M}$ coincide.

The following property will be used frequently. Let $\varphi: X \rightarrow Y$ be an $(r+1)-$ block conjugacy between the mixing MS $X$ and $Y$. It is straightforward that $\varphi$ is 
left resolving. That is, if $\varphi\left(w_{0} \ldots w_{r}\right)=a \in L_{1}(Y)$ and $a^{\prime} \in P_{Y}(a)$, then there is a unique $b \in P_{X}\left(w_{0}\right)$ satisfying $\varphi\left(b w_{0} \ldots w_{r-1}\right)=a^{\prime}$. Therefore there is a one-to-one relation between $P_{Y}(a)$ and $P_{X}\left(w_{0}\right)$. Left resolving maps are onto.

2.2 State splitting and amalgamation. The ideas in this section originated in the work of R.F. Williams [Wi]. The properties that we describe can be found in $[\mathrm{Wi}],[\mathrm{BFK}]$ or $[\mathrm{LM}]$.

Let $M$ be an $n \times n$ nonnegative integral matrix and $G_{M}$ be its associated graph. Let $V_{M}$ and $E_{M}$ be the set of vertices and edges of $G_{M}$ respectively.

We describe an elementary splitting of $G_{M}$. Fix $v \in V_{M}$ and partition the set of edges starting at the vertex $v$ into two nonempty disjoint sets $\alpha^{1}$ and $\alpha^{2}$. We construct a new graph $\bar{G}$ based on this partition as follows.

The vertex set of $\bar{G}$ is $\bar{V}=\left(V_{M} \backslash\{v\}\right) \cup\left\{v_{1}, v_{2}\right\}$. For each edge $e \in \alpha^{i}$, where $i \in\{1,2\}$, put an edge in $\bar{G}$ from $v_{i}$ to the terminal vertex of $e$ in $G_{M}$ if it is not $v$, and draw edges from $v_{i}$ to $v_{1}$ and $v_{2}$ otherwise. For each $e \in E_{M}$ starting at some vertex $\bar{v}, \bar{v} \neq v$, and ending at $v$ put two edges in $\bar{G}$, one of them from $\bar{v}$ to $v_{1}$ and the other one from $\bar{v}$ to $v_{2}$. The other edges in $G_{M}$ remain identically in $\bar{G}$, completing its construction. Denote by $\bar{M}$ the nonnegative integral matrix defining $\bar{G} \cdot \bar{M}$ is called an elementary splitting of $M$, and $M$ an elementary amalgamation of $\bar{M}$. The matrix $\bar{M}$ has a repeated column, the one corresponding to $v_{1}$ and $v_{2}$. To obtain $M$ from $\bar{M}$ it is enough to eliminate one of these columns and add the rows corresponding to $v_{1}$ and $v_{2}$, and index the new rows and columns in the natural way. We notice that if $M$ is a $0-1$ matrix the former process reduces to split $G_{M}$ following partitions of the vertices.

Given a nonnegative integral matrix $M$, there is another nonnegative integral matrix $M_{t}$ with no repeated columns that can be obtained from $M$ after a finite number of elementary amalgamations (see [Wi]). Also, it can be proved (see Lemma 2.7 in $[\mathrm{BFK}])$ that $M_{t}$ is unique up to conjugacy by a permutation matrix. $M_{t}$ is called a total amalgamation of $M$.

If $M$ is an elementary amalgamation of $\bar{M}$ then there is a one-block conjugacy $\varphi: X_{\bar{M}} \rightarrow X_{M}$ with a two-block inverse $\varphi^{-1}$ (see Theorem 4.10 in [LM]); therefore $X_{M}$ and $X_{M_{t}}$ are conjugate by a one-block map $\psi: X_{M} \rightarrow X_{M_{t}}$.

\section{The Result}

Let $X_{0} \subseteq X$ be two nested mixing SFT. We know that $X$ is conjugate to a mixing MS $X_{M}$ given by a nonnegative integral matrix $M$. Let $M_{t}$ be a total amalgamation of $M$, which is unique up to permutation; therefore $X$ is also conjugate to $X_{M_{t}}$. Fix one such representation and denote by $\hat{X}_{M_{t}}$ the associated totally amalgamated system, by $\phi: X \rightarrow \hat{X}_{M_{t}}$ the conjugacy, and put $\hat{X}_{0}=\phi\left(X_{0}\right)$. Observe that $\hat{X}_{0}$ is an SFT but it is not necessarily an MS.

Our main result is the following one.

Theorem 3.1. Let $X_{0} \subseteq X$ be nontrivial mixing SFT. Then, there is a conjugacy $\pi: Y \rightarrow X$, where $Y$ and $Y_{0}=\pi^{-1}\left(X_{0}\right)$ are mixing $M S$ verifying that the sets of letters effectively used by them are the same $\left(L_{1}\left(Y_{0}\right)=L_{1}(Y)\right)$ if and only if for any $a \in L_{1}\left(\hat{X}_{M_{t}}\right)$ there is an $a^{\prime} \in L_{1}\left(\hat{X}_{0}\right)$ such that $P_{\hat{X}_{M_{t}}}(a)=P_{\hat{X}_{M_{t}}}\left(a^{\prime}\right)$.

First, we supply two examples to illustrate how the conditions of the theorem apply. In the first one we present a pair of SFT $\left(X, X_{0}\right)$ which fails to meet the condition of Theorem 3.1 and therefore the conclusion of the theorem does not 
hold. In the second example we exhibit a pair of SFT meeting the condition of the theorem, and we provide the corresponding conjugate MS and the conjugacy.

Example 1. Consider the mixing one-sided Markov shift $X \subseteq\{0,1,2\}$ such that the blocks 11, 12 and 21 are not allowed, and consider a subshift of finite type $X_{0}$ in $X$ using the alphabet $\{0,1\}$. This example fails to meet the condition of Theorem 3.1. In fact, the matrix which defines $X$ is already totally amalgamated and for any pair of distinct letters $a, b \in\{0,1,2\}$ there is $c \in\{0,1,2\}$ such that $c a$ is a block in $X$ but $c b$ is not an allowed block in $X$, or vice versa. As Theorem 3.1 says, it is impossible to recode this pair of systems as an MS, where the symbols used to describe the recoded version of $X_{0}$ are all the symbols used to describe the recoded version of $X$. Let us give an argument for that. If we suppose there are Markov shifts $Y$ and $Y_{0}$ with $Y_{0} \subseteq Y$ using exactly the same letters, and a conjugacy $\varphi: Y \rightarrow X$ that maps $Y_{0}$ onto $X_{0}$, then by a recursive argument it is possible to prove that $\varphi$ is a letter to letter projection, that is, a 1-block map. Therefore $X_{0}$ and $X$ use exactly the same alphabet, which is a contradiction.

Example 2. Let $X \subseteq\{0,1,2\}^{\mathbb{N}}$ be the MS forbidding the two-blocks 11,12 and 20 , and $X_{0} \subseteq\{0,1\}^{\mathbb{N}}$ be the MS such that $11 \notin L_{2}\left(X_{0}\right)$. Clearly $X_{0} \subseteq X$ and $L_{1}(X) \neq L_{1}\left(X_{0}\right)$. Also, the letter $2 \in L_{1}(X) \backslash L_{1}\left(X_{0}\right)$ has the same predecessor set as $1 \in L_{1}\left(X_{0}\right)$. This property is stronger than the condition of Theorem 3.1, as is proved below. On the other hand, it is not difficult to see that the matrix [2] is a total amalgamation for $X$, and then the condition of Theorem 3.1 is trivially satisfied.

Now we define Markov shifts $Y_{0}$ and $Y$, where $Y_{0} \subseteq Y$ and $L_{1}\left(Y_{0}\right)=L_{1}(Y)$, and a conjugacy $\varphi: Y \rightarrow X$ such that $\varphi\left(Y_{0}\right)=X_{0}$. The MS $Y$ is defined in $\{0,1,2\}^{\mathbb{N}}$ by forbidding the two-blocks 01,20 and 22 , and $Y_{0}$ is the MS in $Y$ such that $11 \notin L_{2}\left(Y_{0}\right)$. Clearly $L_{1}\left(Y_{0}\right)=L_{1}(Y)$. The conjugacy $\varphi: Y \rightarrow X$ has the following block code: $\varphi(00)=0, \varphi(02)=0, \varphi(21)=0, \varphi(10)=1, \varphi(12)=1$, $\varphi(11)=2$. A simple computation yields $\varphi\left(Y_{0}\right)=X_{0}$. The MS $Y_{0}$ is obtained after state splitting of $X_{0}$ with respect to the symbol 0 , and $\varphi$ is an extension of the resulting map to $Y$.

The proof of the theorem will follow once we have proved some lemmas and propositions. The following property will be useful. Let $Z$ and $W$ be subshifts with $W \subseteq Z$. The couple $(Z, W)$ is said to satisfy property $(N)$ if

$(N) \quad$ for every $b \in L_{1}(Z)$ there exists $b^{\prime} \in L_{1}(W)$ such that $P_{Z}(b)=P_{Z}\left(b^{\prime}\right)$.

Let us begin by showing that the condition of the theorem is necessary. For this we assume there is a conjugacy $\pi: Y \rightarrow X$, where $Y$ and $Y_{0}=\pi^{-1}\left(X_{0}\right)$ are mixing MS with $L_{1}\left(Y_{0}\right)=L_{1}(Y)$.

Let $M^{\prime}$ be the $0-1$ matrix defining $Y, Y_{M^{\prime}}$ be the arc representation of $Y$, and $Y_{0}^{\prime}$ be the arc representation of $Y_{0}$. The pair $\left(Y_{M^{\prime}}, Y_{0}^{\prime}\right)$ satisfies property $(N)$.

Since $X$ is conjugate with $\hat{X}_{M_{t}}$, we can construct a conjugacy $\pi^{\prime}: Y_{M^{\prime}} \rightarrow \hat{X}_{M_{t}}$ with $\pi^{\prime}\left(Y_{0}^{\prime}\right)=\hat{X}_{0}$. Hence, by William's theorem (see Theorem 2.11 in [BFK]), $M^{\prime}$ and $M_{t}$ have the same total amalgamations. In particular, $M_{t}$ is a total amalgamation of $M^{\prime}$. Let $\pi^{\prime \prime}$ be a conjugacy from $Y_{M^{\prime}}$ to $\hat{X}_{M_{t}}$ compatible with a sequence of elementary amalgamations from $M^{\prime}$ to $M_{t}$, and put $\hat{Y}_{0}=\pi^{\prime \prime}\left(Y_{0}^{\prime}\right)$. The maps $\pi^{\prime \prime}$ and $\pi^{\prime}$ induce an automorphism $\varphi: \hat{X}_{M_{t}} \rightarrow \hat{X}_{M_{t}}$, where $\varphi\left(\hat{Y}_{0}\right)=\hat{X}_{0}$. 
Therefore, to state the necessary condition it is enough to prove that property $(N)$ can be transferred from $\left(Y_{M^{\prime}}, Y_{0}^{\prime}\right)$ to $\left(\hat{X}_{M_{t}}, \hat{Y}_{0}\right)$ and from $\left(\hat{X}_{M_{t}}, \hat{Y}_{0}\right)$ to $\left(\hat{X}_{M_{t}}, \hat{X}_{0}\right)$.

Lemma 3.2. Let $\psi: X_{M} \rightarrow X_{M^{\prime}}$ be a one-block conjugacy between the MS defined by the nonnegative integral matrices $M$ and $M^{\prime}$, and let $X_{0}$ be a subshift contained in $X_{M}$. Then $\left(X_{M^{\prime}}, \psi\left(X_{0}\right)\right)$ has property $(N)$ whenever $\left(X_{M}, X_{0}\right)$ does.

Proof. Let $b \in L_{1}\left(X_{M^{\prime}}\right)$ and take $y \in X_{M^{\prime}}$ such that $b y \in X_{M^{\prime}}$. Since $\psi$ is a conjugacy, there are unique $a \in L_{1}\left(X_{M}\right)$ and $x \in X_{M}$ with $\psi(a x)=b y$. Fix $a^{\prime} \in$ $L_{1}\left(X_{0}\right)$ which satisfies $P_{X_{M}}(a)=P_{X_{M}}\left(a^{\prime}\right)$, and take $x_{0} \in X_{0}$ such that $a^{\prime} x_{0} \in X_{0}$. We will prove that $P_{X_{M^{\prime}}}(b)=P_{X_{M^{\prime}}}\left(b^{\prime}\right)$, where $b^{\prime}$ satisfies $\psi\left(a^{\prime} x_{0}\right)=b^{\prime} y_{0} \in \psi\left(X_{0}\right)$.

Let $c \in P_{X_{M^{\prime}}}(b)$. The point cby is in $X_{M^{\prime}}$ and it has a unique preimage by $\psi$, $d a x \in X_{M}$. This implies $d \in P_{X_{M}}(a)=P_{X_{M}}\left(a^{\prime}\right)$ and $d a^{\prime} x_{0} \in X_{M}$. Therefore, $\psi\left(d a^{\prime} x_{0}\right)=\psi(d) \psi\left(a^{\prime} x_{0}\right)=c b^{\prime} y_{0}$ (recall that $\psi$ is one-block), which means $c \in$ $P_{X_{M^{\prime}}}\left(b^{\prime}\right)$. We have proved $P_{X_{M^{\prime}}}(b) \subseteq P_{X_{M^{\prime}}}\left(b^{\prime}\right)$. The other inclusion is shown in an analogous way.

The conjugacy $\pi^{\prime \prime}$ between $Y_{M^{\prime}}$ and $\hat{X}_{M_{t}}$ is constructed as a composition of elementary amalgamations; then it is one-block. Therefore, by the last lemma, the pair $\left(\hat{X}_{M_{t}}, \hat{Y}_{0}\right)$ satisfies property $(N)$.

To show that $\varphi$ preserves property $(N)$ it is necessary to go further into the structure of the automorphisms of one-sided MS. We will rely heavily on the results in $[\mathrm{BFK}]$.

Lemma 3.3. $\left(\hat{X}_{M_{t}}, \hat{X}_{0}\right)$ has property $(N)$.

Proof. From Theorem 2.12 in [BFK] it follows that the automorphism $\varphi: \hat{X}_{M_{t}} \rightarrow$ $\hat{X}_{M_{t}}$ can be decomposed as $\varphi=\varphi_{0} \circ \varphi_{1}$, where $\varphi_{1}$ is a composition of simple automorphisms of $\hat{X}_{M_{t}}$ and $\varphi_{0}$ is a vertex automorphism of $\hat{X}_{M_{t}}$. In particular, $\varphi_{0}$ is a one-block automorphism (definitions of simple and vertex automorphisms can be found in $[\mathrm{BFK}])$.

On the other hand, from Lemma 2.23 in $[\mathrm{BFK}]$ it follows that $\varphi_{1}$ satisfies the following property: for all $x \in \hat{X}_{M_{t}}$ and $i \in \mathbb{N},\left(\varphi_{1}(x)\right)_{i}$ has the same initial and terminal vertex as $x_{i}$ in $G_{M_{t}}$. Therefore, since $\left(\hat{X}_{M_{t}}, \hat{Y}_{0}\right)$ satisfies property $(N)$, for any vertex in $G_{M_{t}}$ there is a point in $\varphi_{1}\left(\hat{Y}_{0}\right)$ starting from it, that is, $\left(\hat{X}_{M_{t}}, \varphi_{1}\left(\hat{Y}_{0}\right)\right)$ verifies property $(N)$.

To conclude the proof, observe that $\varphi_{0}$ is a one-block automorphism and $\varphi_{0} \circ$ $\varphi_{1}\left(\hat{Y}_{0}\right)=\hat{X}_{0}$, so Lemma 3.2 implies that $\left(\hat{X}_{M_{t}}, \hat{X}_{0}\right)$ also verifies the property.

We have proved the necessity of the condition of Theorem 3.1. The proof of the sufficiency is more involved. To do it we use state splitting.

Lemma 3.4. Let $W_{0} \subseteq W$ be nontrivial mixing $M S$ such that $L_{1}\left(W_{0}\right)=L_{1}(W)$ and let $w \in L_{1}(W)$. Then, there are mixing $M S \quad \bar{W}_{0} \subseteq \bar{W}$, where $L_{1}\left(\bar{W}_{0}\right)=$ $L_{1}(\bar{W})$, and a one-block conjugacy $\varphi: \bar{W} \rightarrow W$ with $\varphi\left(\bar{W}_{0}\right)=W_{0}$ such that $\varphi^{-1}(\{w\})=\left\{w_{1}, w_{2}\right\}, P_{\bar{W}}\left(w_{1}\right)=P_{\bar{W}}\left(w_{2}\right)$ and $S_{\bar{W}}\left(w_{1}\right) \cap S_{\bar{W}}\left(w_{2}\right)=\varnothing$.

Proof. Since $W_{0}$ and $W$ are mixing MS using more than one letter, there are states $u_{0}=w, u_{1}, \ldots, u_{s} \in L_{1}(W)=L_{1}\left(W_{0}\right)$ such that (i) $S_{W_{0}}\left(u_{i}\right)=\left\{u_{i+1}\right\}$ for $i \in$ $\{0, \ldots, s-1\}$, (ii) $\left|S_{W_{0}}\left(u_{s}\right)\right| \geq 2$ and (iii) $u_{i} \neq u_{j}$ if $i \neq j$. 
Now in $W$ we split the state $u_{s}$ into $u_{s}^{(1)}$ and $u_{s}^{(2)}$, following a partition $\mathcal{P}=$ $\left\{\alpha^{(1)}, \alpha^{(2)}\right\}$ of $S_{W}\left(u_{s}\right)$ such that $\alpha^{(1)} \cap S_{W_{0}}\left(u_{s}\right) \neq \varnothing$ and $\alpha^{(2)} \cap S_{W_{0}}\left(u_{s}\right) \neq \varnothing$. This is possible because $\left|S_{W_{0}}\left(u_{s}\right)\right| \geq 2$. Denote by $W^{(s)}$ the resulting mixing MS and by $\varphi^{(s)}: W^{(s)} \rightarrow W$ the corresponding amalgamation map (recall that it is one-block). We have that $L_{1}\left(W^{(s)}\right)=\left(L_{1}(W) \backslash\left\{u_{s}\right\}\right) \cup\left\{u_{s}^{(1)}, u_{s}^{(2)}\right\}$, and, since both atoms of $\mathcal{P}$ intersect $S_{W_{0}}\left(u_{s}\right), L_{1}\left(W_{0}^{(s)}\right)=L_{1}\left(W^{(s)}\right)$, where $\varphi^{(s)}\left(W_{0}^{(s)}\right)=W_{0}$. Observe that $W_{0}^{(s)}$ is also a mixing MS.

In this new system $S_{W_{0}^{(s)}}\left(u_{s-1}\right)=\left\{u_{s}^{(1)}, u_{s}^{(2)}\right\}$ and $S_{W_{0}^{(s)}}\left(u_{i}\right)=\left\{u_{i+1}\right\}, i \in\{0, \ldots$, $s-2\}$. Thus, we can repeat the last procedure recursively until we reach state $w$. We obtain nontrivial mixing MS $W_{0}^{(i)} \subseteq W^{(i)}$ with $L_{1}\left(W^{(i)}\right)=L_{1}\left(W_{0}^{(i)}\right)$ and one-block conjugacies $\varphi^{(i)}: W^{(i)} \rightarrow W^{(i+1)}$ such that $\varphi^{(i)}\left(W_{0}^{(i)}\right)=W_{0}^{(i+1)}$, for $i \in\{0, \ldots, s\}$ (we have put $W^{(s+1)}=W$ and $W_{0}^{(s+1)}=W_{0}$ ). Moreover, in $W^{(1)}$ the state $w$ is split into the states $w_{1}$ and $w_{2}$, ensuring that $P_{W^{(0)}}\left(w_{1}\right)=P_{W^{(0)}}\left(w_{2}\right)$ and $S_{W^{(0)}}\left(w_{1}\right) \cap S_{W^{(0)}}\left(w_{2}\right)=\varnothing$.

We conclude by defining $\bar{W}=W^{(0)}, \bar{W}_{0}=W_{0}^{(0)}$, and $\varphi: \bar{W} \rightarrow W$ as the composition of the one-block maps $\varphi^{(i)}$.

Let $W, W_{0}, Z, \bar{Z}$ be nontrivial mixing MS such that $W_{0} \subseteq W, Z \subseteq \bar{Z}$, and let $\pi: W \rightarrow Z$ be a two-block conjugacy. Let us point out that, since $\pi$ is left resolving, we have $P_{Z}\left(\pi\left(v v^{\prime \prime}\right)\right)=P_{Z}\left(\pi\left(v v^{\prime}\right)\right)$ for $v v^{\prime}, v v^{\prime \prime} \in L_{2}(W)$. Now we define a property which strengthens this last one. We say that the 5 -tuple $\left(\pi, W_{0}, W, Z, \bar{Z}\right)$ satisfies property $(P)$ if the following conditions hold:

$\left(P_{1}\right)$ for any $v v^{\prime \prime}, v v^{\prime} \in L_{2}(W) ; P_{\bar{Z}}\left(\pi\left(v v^{\prime \prime}\right)\right)=P_{\bar{Z}}\left(\pi\left(v v^{\prime}\right)\right)$;

$\left(P_{2}\right) L_{1}\left(W_{0}\right)=L_{1}(W)$.

When $\pi$ is a one-block map condition $\left(P_{1}\right)$ follows directly.

For any $a \in L_{1}(Z)$ define the MS $Z_{a}$ such that $L_{2}\left(Z_{a}\right)=L_{2}(Z) \cup\left\{a b \in L_{2}(\bar{Z}) \mid b \in\right.$ $\left.L_{1}(Z)\right\}$. Notice that $Z_{a}$ is also a nontrivial mixing MS contained in $\bar{Z}$ which contains $Z$.

The following proposition will be used in the one-block version. For the sake of completeness we give a more general statement.

Proposition 3.5. Let $\left(\pi, W_{0}, W, Z, \bar{Z}\right)$ be a 5-tuple with property $(P)$ and a $\in$ $L_{1}(Z)$. Then there are mixing $M S W_{0}^{\prime} \subseteq W^{\prime}$ and a two-block conjugacy $\pi^{\prime}: W^{\prime} \rightarrow$ $Z_{a}$ such that $\pi^{\prime}\left(W_{0}^{\prime}\right)=\pi\left(W_{0}\right)$ and the 5-tuple $\left(\pi^{\prime}, W_{0}^{\prime}, W^{\prime}, Z_{a}, \bar{Z}\right)$ has property $(P)$. Moreover, $\pi^{\prime}$ is one-block whenever $\pi$ is one-block.

Proof. Let us fix a state $w \in L_{1}(W)$ such that $\pi\left(w w^{\prime}\right)=a$ for some $w^{\prime} \in L_{1}(W)$. This choice is possible because $a \in L_{1}(Z)$ and $\pi$ is onto.

We apply Lemma 3.4 to $w, W_{0}$ and $W$ in order to produce nontrivial mixing MS $\bar{W}$ and $\bar{W}_{0}$ satisfying $\bar{W}_{0} \subseteq \bar{W}$ and $L_{1}(\bar{W})=L_{1}\left(\bar{W}_{0}\right)$, and a one-block conjugacy $\varphi: \bar{W} \rightarrow W$ such that $\varphi\left(\bar{W}_{0}\right)=W_{0}$ and $\varphi^{-1}(\{w\})=\left\{w_{1}, w_{2}\right\}$ with $P_{\bar{W}}\left(w_{1}\right)=$ $P_{\bar{W}}\left(w_{2}\right), S_{\bar{W}}\left(w_{1}\right) \cap S_{\bar{W}}\left(w_{2}\right)=\varnothing$. Set $\bar{\pi}=\pi \circ \varphi$; then it follows directly that the 5 -tuple $\left(\bar{\pi}, \bar{W}_{0}, \bar{W}, Z, \bar{Z}\right)$ satisfies property $(P)$ and $\bar{\pi}\left(\bar{W}_{0}\right)=\pi\left(W_{0}\right)$. Notice that if $\pi$ is a one-block map then $\bar{\pi}$ is also one-block, with $\bar{\pi}\left(w_{1}\right)=\bar{\pi}\left(w_{2}\right)=\pi(w)=a$.

The following property $(Q)$ can also be deduced from this construction:

$\left(Q_{1}\right)$ if $b=\bar{\pi}\left(v v^{\prime}\right)$ and $b^{\prime}=\bar{\pi}\left(v v^{\prime \prime}\right)$ with $v v^{\prime}, v v^{\prime \prime} \in L_{2}(\bar{W})$, then $P_{Z_{a}}(b)=$ $P_{Z_{a}}\left(b^{\prime}\right)$

$\left(Q_{2}\right)$ under the same conditions as in $\left(Q_{1}\right)$ and $v=w_{1}$ or $v=w_{2}$ we have $P_{Z_{a}}(b)=P_{Z_{a}}\left(b^{\prime}\right)=P_{Z_{a}}(a)$. 
In fact, from property $(P)$ we get $P_{\bar{Z}}(b)=P_{\bar{Z}}\left(b^{\prime}\right)$ and $P_{Z}(b)=P_{Z}\left(b^{\prime}\right)$, and since $\varphi$ is one-block, $P_{\bar{Z}}(b)=P_{\bar{Z}}\left(b^{\prime}\right)=P_{\bar{Z}}(a)$ and $P_{Z}(b)=P_{Z}\left(b^{\prime}\right)=P_{Z}(a)$ whenever $v=w_{1}$ or $v=w_{2}$, which proves the desired property.

The idea of the proof is to extend $\bar{\pi}: \bar{W} \rightarrow Z$ to a factor map whose domain is an MS $W^{\prime}$ containing $\bar{W}$ and whose range is $Z_{a}$. In that purpose consider the sets of letters,

$$
\begin{aligned}
& \mathcal{B}=\left\{b \in L_{1}(Z) \mid a b \in L_{2}\left(Z_{a}\right) \backslash L_{2}(Z)\right\}, \\
& \mathcal{U}=\left\{u \in L_{1}(\bar{W}) \mid \exists u^{\prime} \in S_{\bar{W}}(u), \bar{\pi}\left(u u^{\prime}\right) \in \mathcal{B}\right\} .
\end{aligned}
$$

From property $(Q)$ we deduce that,

$$
\text { if } u \in \mathcal{U} \text { and } u^{\prime} \in S_{\bar{W}}(u) \text {, then } \bar{\pi}\left(u u^{\prime}\right) \in \mathcal{B} \text {. }
$$

We define the Markov shift $W^{\prime}$ through the set of two blocks

$$
L_{2}\left(W^{\prime}\right) \doteq L_{2}(\bar{W}) \cup\left\{w_{1} u \mid u \in \mathcal{U}, w_{1} u \notin L_{2}(\bar{W})\right\} \cup\left\{w_{2} u \mid u \in \mathcal{U}, w_{1} u \in L_{2}(\bar{W})\right\} .
$$

By construction, $S_{\bar{W}}\left(w_{1}\right) \cap S_{\bar{W}}\left(w_{2}\right)=\varnothing$; then $w_{2} u \notin L_{2}(\bar{W})$ whenever $w_{1} u \in$ $L_{2}(\bar{W})$. Therefore for all $u \in \mathcal{U}$ there is a unique $w_{i}, i \in\{1,2\}$, such that $w_{i} u \in$ $L_{2}\left(W^{\prime}\right)$ and $w_{i} u \notin L_{2}(\bar{W})$. Also $W^{\prime}$ is a mixing MS.

We extend $\bar{\pi}$ to $W^{\prime}$ by putting $\pi^{\prime}\left(w_{i} u\right)=a$ if $w_{i} u \in L_{2}\left(W^{\prime}\right) \backslash L_{2}(\bar{W}), i \in\{1,2\}$, and $\pi^{\prime}(u v)=\bar{\pi}(u v)$ if $u v \in L_{2}(\bar{W})$. Set $W_{0}^{\prime}=\bar{W}_{0}$; then $\pi^{\prime}\left(W_{0}^{\prime}\right)=\bar{\pi}\left(\bar{W}_{0}\right)$. By construction, if $\bar{\pi}$ is one-block the resulting $\pi^{\prime}$ is also one-block because $\bar{\pi}\left(w_{1}\right)=$ $\bar{\pi}\left(w_{2}\right)=a$. In the sequel we shall prove that $\pi^{\prime}$ is a conjugacy between the mixing MS $W^{\prime}$ and $Z_{a}$. We begin by showing that $\pi^{\prime}$ is a left resolving factor map onto $Z_{a}$.

Fix $v \in L_{1}\left(W^{\prime}\right)=L_{1}(\bar{W})$. From the construction it follows that $P_{W^{\prime}}(v)$ is equal to $P_{\bar{W}}(v)$ when $v \notin \mathcal{U}$, and $P_{W^{\prime}}(v)$ is equal to $P_{\bar{W}}(v) \cup\left\{w_{i}\right\}$ for a unique $i \in\{1,2\}$ when $v \in \mathcal{U}$. Take $b \in L_{1}\left(Z_{a}\right)=L_{1}(Z)$ such that $\pi^{\prime}(v \bar{v})=b$ for some $\bar{v} \in S_{\bar{W}}(v)$. The left resolving property of $\bar{\pi}$ implies that $P_{\bar{W}}(v)$ and $P_{Z}(b)$ are in a one-to-one correspondence and $\left\{\bar{\pi}\left(v^{\prime} v\right) \mid v^{\prime} \in P_{\bar{W}}(v)\right\}=P_{Z}(b)$. Also, for any $v^{\prime} \in P_{\bar{W}}(v)$, $\pi^{\prime}\left(v^{\prime} v\right) b=\bar{\pi}\left(v^{\prime} v\right) \bar{\pi}(v \bar{v}) \in L_{2}(Z) \subseteq L_{2}\left(Z_{a}\right)$. In addition, if $v \in \mathcal{U}$ then $b \in \mathcal{B}$, and there is a unique $v^{\prime} \in P_{W^{\prime}}(v)$ that is equal to $w_{1}$ or $w_{2}$ such that $\pi^{\prime}\left(v^{\prime} v\right)=a$. Hence $a b \in L_{2}\left(Z_{a}\right)$. We conclude that for any $b^{\prime \prime} \in P_{Z_{a}}(b)$ there is a unique $v^{\prime} \in P_{W^{\prime}}(v)$ such that $\pi^{\prime}\left(v^{\prime} v\right)=b^{\prime \prime}$ and $\left\{\pi^{\prime}\left(v^{\prime} v\right) b \mid v^{\prime} \in P_{W^{\prime}}(v)\right\} \subseteq L_{2}\left(Z_{a}\right)$.

Now suppose that $v=w_{i}$ for some $i \in\{1,2\}$ and $b=\pi^{\prime}\left(w_{i} \bar{v}\right)$ with $w_{i} \bar{v} \in$ $L_{2}\left(W^{\prime}\right) \backslash L_{2}(\bar{W})$; then $b$ must be equal to $a$. We know from $(Q)$ that $P_{Z_{a}}(a)=$ $P_{Z_{a}}\left(b^{\prime}\right)$ for any $b^{\prime}=\bar{\pi}\left(v v^{\prime}\right)$ with $v v^{\prime} \in L_{2}(\bar{W})$. Then for any $b^{\prime \prime} \in P_{Z_{a}}(a)$ there is a unique $v^{\prime \prime} \in P_{W^{\prime}}\left(w_{i}\right)$ such that $\pi^{\prime}\left(v^{\prime \prime} v\right)=b^{\prime \prime}$ and $\left\{\pi^{\prime}\left(v^{\prime \prime} v\right) a \mid v^{\prime \prime} \in P_{W^{\prime}}(v)\right\} \subseteq$ $L_{2}\left(Z_{a}\right)$. Therefore $\pi^{\prime}$ is a left resolving factor map from $W^{\prime}$ to $Z_{a}$.

Now we will prove that $\pi^{\prime}$ is one-to-one. Let $x \in Z_{a}$. If $x \in Z$, there is a unique $y \in \bar{W}$ such that $\bar{\pi}(y)=\pi^{\prime}(y)=x$. If we can find another $y^{\prime} \in W^{\prime}$ such that $\pi^{\prime}\left(y^{\prime}\right)=x$ it must be in $W^{\prime} \backslash \bar{W}$, and, for some integer $i^{*}, x_{i^{*}} x_{i^{*}+1}=$ $a x_{i^{*}+1} \notin L_{2}(Z)$, which is a contradiction. Then $x \in Z$ has a unique preimage by $\pi^{\prime}$. Furthermore, since $\pi^{\prime}$ is left resolving, we can extend this last property to the points $x=z x^{\prime} \in Z_{a}$ such that $z \in L\left(Z_{a}\right)$ and $x^{\prime} \in Z$. In fact, the unique preimage of $x$ is $y=z^{\prime} y^{\prime}$, where $\left|z^{\prime}\right|=|z|, \quad \bar{\pi}\left(y^{\prime}\right)=x^{\prime}$, and $z^{\prime}$ is uniquely determined from $z, x^{\prime}$ and $y^{\prime}$ by using the left resolving property.

It remains to consider the case when $x \in Z_{a}$ and for infinitely many coordinates we have $x_{i} x_{i+1}=a b \in L_{2}\left(Z_{a}\right) \backslash L_{2}(Z)$. If $y \in W^{\prime}$ is a preimage of such a $x$, then $y_{i} \in\left\{w_{1}, w_{2}\right\}$ whenever $x_{i} x_{i+1} \in L_{2}\left(Z_{a}\right) \backslash L_{2}(Z)$. Fix a coordinate $j \geq 2$ such that 
$x_{j} x_{j+1} \notin L_{2}(Z)$. We have that $w_{1} \in \mathcal{U}$ if and only if $w_{2} \in \mathcal{U}$. In fact, from property $(Q)$ we have that $a \bar{\pi}\left(w_{1} v\right) \in L_{2}(\bar{Z}) \backslash L_{2}(Z)$ if $w_{1} \in \mathcal{U}$ and $v \in S_{\bar{W}}\left(w_{1}\right)$. Again by using property $(Q)$ we obtain that, for $\bar{v} \in S_{\bar{W}}\left(w_{2}\right), P_{\bar{Z}}\left(\bar{\pi}\left(w_{1} v\right)\right)=P_{\bar{Z}}\left(\bar{\pi}\left(w_{2} \bar{v}\right)\right)=$ $P_{\bar{Z}}(a)$ and $P_{Z}\left(\bar{\pi}\left(w_{1} v\right)\right)=P_{Z}\left(\bar{\pi}\left(w_{2} \bar{v}\right)\right)=P_{Z}(a)$. Then $w_{2} \in \mathcal{U}$. Therefore, there is at most one $j \in\{1,2\}$ such that $w_{j} w_{1}, w_{j} w_{2} \in L_{2}\left(W^{\prime}\right) \backslash L_{2}(\bar{W})$. It follows that $P_{W^{\prime}}\left(w_{1}\right)=P_{W^{\prime}}\left(w_{2}\right)$ and also $\pi^{\prime}\left(\bar{v} w_{1}\right)=\pi^{\prime}\left(\bar{v} w_{2}\right)$ for any $\bar{v} \in P_{W^{\prime}}\left(w_{1}\right)$. Therefore, for any $c \in P_{Z_{a}}(a)$ there is a unique $\bar{v} \in P_{W^{\prime}}\left(w_{1}\right)$ such that $\pi^{\prime}\left(\bar{v} w_{1}\right)=\pi^{\prime}\left(\bar{v} w_{2}\right)=c$. This implies that the value of $y_{j-1}$ is uniquely determined, and therefore, for all $i<j, y_{i}$ is determined. Thus, applying the last argument for infinitely many $j$, we find a unique $y \in W^{\prime}$ such that $\pi^{\prime}(y)=x$. We have proved that $\pi^{\prime}$ is a two-block conjugacy between the mixing MS $W^{\prime}$ and $Z_{a}$.

To conclude we show that $\left(\pi^{\prime}, W_{0}^{\prime}, W^{\prime}, Z_{a}, \bar{Z}\right)$ has property $(P)$. We have already mentioned that $W_{0}^{\prime}$ and $W^{\prime}$ are nontrivial mixing MS with $W_{0}^{\prime} \subseteq W^{\prime}, L_{1}\left(W_{0}^{\prime}\right)=$ $L_{1}\left(W^{\prime}\right)$, and $\pi^{\prime}: W^{\prime} \rightarrow Z_{a}$ is a two-block conjugacy which is one-block if $\pi$ is oneblock. Furthermore, since $W_{0}^{\prime}=\bar{W}_{0}$ we also have the property $\pi^{\prime}\left(W_{0}^{\prime}\right)=\bar{\pi}\left(\bar{W}_{0}\right)=$ $\pi\left(W_{0}\right)$.

Finally, to establish property $\left(P_{1}\right)$ it is enough to observe that for $w_{i} v \in L_{2}\left(W^{\prime}\right) \backslash$ $L_{2}(\bar{W}), i \in\{1,2\}$, the letter $\pi^{\prime}\left(w_{i} v\right)=a$ has the same predecessor set in $\bar{Z}$ as any other symbol $a^{\prime} \in L_{1}(Z)$ such that $\bar{\pi}\left(w_{i} \bar{v}\right)=a^{\prime}$ for $w_{i} \bar{v} \in L_{2}(\bar{W})$, because $\left(\bar{\pi}, \bar{W}, \bar{W}_{0}, Z, \bar{Z}\right)$ has property $(P)$.

Corollary 3.6. Let $\left(\bar{\pi}, \bar{W}_{0}, \bar{W}, Z, \bar{Z}\right)$ be a 5-tuple with property $(P)$ such that $L_{1}(Z)$ $=L_{1}(\bar{Z})$. Then there is a 5-tuple $\left(\pi, W_{0}, W, \bar{Z}, \bar{Z}\right)$ with property $(P)$ such that $\pi\left(W_{0}\right)=\bar{\pi}\left(\bar{W}_{0}\right)$. If $\bar{\pi}$ is one-block then $\pi$ is also one-block.

Proof. Put $L_{1}(Z)=\left\{a_{1}, \ldots, a_{s}\right\}$. By applying recursively Proposition 3.5 we prove the existence of 5 -tuples $\left(\pi^{(i)}, W_{0}^{(i)}, W^{(i)}, Z^{(i)}, \bar{Z}\right)$ with property $(P)$, where $Z^{(i)}=$ $\left(Z^{(i-1)}\right)_{a_{i}}, Z^{(0)}=Z$ and $\pi^{(i)}\left(W_{0}^{(i)}\right)=\bar{\pi}\left(\bar{W}_{0}\right)$. Therefore, $\left(\pi^{(s)}, W_{0}^{(s)}, W^{(s)}, Z^{(s)}=\right.$ $\bar{Z}, \bar{Z})$ satisfies the requirements of the corollary.

Let $\pi: W \rightarrow Z$ be a two-block conjugacy between the nontrivial mixing MS $W, Z$, and let $W_{0}$ be a mixing MS contained in $W$ such that $L_{1}\left(W_{0}\right)=L_{1}(W)$. Let $\bar{Z}$ be a mixing MS containing $Z$. We will say that the 5 -tuple $\left(\pi, W_{0}, W, Z, \bar{Z}\right)$ has property $\left(P^{*}\right)$ if it satisfies the following additional conditions:

$\left(P_{1}^{*}\right)\left\{a b \in L_{2}(\bar{Z}) \mid a, b \in L_{1}(Z)\right\}=L_{2}(Z)$,

$\left(P_{2}^{*}\right) \forall v v^{\prime \prime}, v v^{\prime} \in L_{2}(W), P_{\bar{Z}}\left(\pi\left(v v^{\prime \prime}\right)\right)=P_{\bar{Z}}\left(\pi\left(v v^{\prime}\right)\right)$,

$\left(P_{3}^{*}\right) \forall a \in L_{1}(\bar{Z}) \quad \exists a^{\prime} \in L_{1}(Z), P_{\bar{Z}}(a)=P_{\bar{Z}}\left(a^{\prime}\right)$.

Let $a \in L_{1}(\bar{Z}) \backslash L_{1}(Z)$ be such that $a c \in L_{2}(\bar{Z})$ for at least one $c \in L_{1}(Z)$ and also assume that $\left(\pi, W_{0}, W, Z, \bar{Z}\right)$ has property $\left(P^{*}\right)$. We can define the MS $Z_{a}^{*}$ such that $L_{1}\left(Z_{a}^{*}\right)=L_{1}(Z) \cup\{a\}$ and $L_{2}\left(Z_{a}^{*}\right)=L_{2}(Z) \cup\left\{b a \in L_{2}(\bar{Z}) \mid b \in L_{1}\left(Z_{a}^{*}\right)\right\} \cup\{a c \in$ $\left.L_{2}(\bar{Z}) \mid c \in L_{1}\left(Z_{a}^{*}\right)\right\}$. It is straightforward that $Z_{a}^{*}$ is also a nontrivial mixing MS. Properties $\left(P_{1}^{*}\right)$ and $\left(P_{3}^{*}\right)$ of $\left(P^{*}\right)$ imply that $\left\{b a \in L_{2}(\bar{Z}) \mid b \in L_{1}\left(Z_{a}^{*}\right)\right\}=\{b a \in$ $\left.L_{2}(\bar{Z}) \mid b \in P_{Z}\left(a^{\prime}\right) \cup\{a\}\right\}$ for any $a^{\prime} \in L_{1}(Z)$ with $P_{\bar{Z}}(a)=P_{\bar{Z}}\left(a^{\prime}\right)$.

Proposition 3.7. Let $\left(\pi, W_{0}, W, Z, \bar{Z}\right)$ be a 5-tuple with property $\left(P^{*}\right)$, and let $a \in L_{1}(\bar{Z}) \backslash L_{1}(Z)$ be such that $a c \in L_{2}(\bar{Z})$ for at least one $c \in L_{1}(Z)$. Then there are mixing $M S W_{0}^{\prime} \subseteq W^{\prime}$ and a two-block conjugacy $\pi^{\prime}: W^{\prime} \rightarrow Z_{a}^{*}$ such that $\pi^{\prime}\left(W_{0}^{\prime}\right)=\pi\left(W_{0}\right)$ and the 5-tuple $\left(\pi^{\prime}, W_{0}^{\prime}, W^{\prime}, Z_{a}^{*}, \bar{Z}\right)$ has property $\left(P^{*}\right)$.

Proof. Fix $a^{\prime} \in L_{1}(Z)$ and $w \in L_{1}(W)$ such that $P_{\bar{Z}}\left(a^{\prime}\right)=P_{\bar{Z}}(a)$ and $\pi\left(w w^{\prime}\right)=a^{\prime}$ for some $w^{\prime} \in L_{1}(W)$. Now apply Lemma 3.4 to $w, W$ and $W_{0}$ in order to produce 
nontrivial mixing MS $\bar{W}$ and $\bar{W}_{0}$, where $\bar{W}_{0} \subseteq \bar{W}$ and $L_{1}(\bar{W})=L_{1}\left(\bar{W}_{0}\right)$, and a one-block conjugacy $\varphi: \bar{W} \rightarrow W$ which satisfies $\varphi\left(\bar{W}_{0}\right)=W_{0}$ and $\varphi^{-1}(\{w\})=$ $\left\{w_{1}, w_{2}\right\}$. We also recall that $P_{\bar{W}}\left(w_{1}\right)=P_{\bar{W}}\left(w_{2}\right)$ and $S_{\bar{W}}\left(w_{1}\right) \cap S_{\bar{W}}\left(w_{2}\right)=\varnothing$. Therefore, the 5 -tuple $\left(\bar{\pi}, \bar{W}_{0}, \bar{W}, Z, \bar{Z}\right)$, where $\bar{\pi}=\pi \circ \varphi$, has property $\left(P^{*}\right)$ and $\bar{\pi}\left(\bar{W}_{0}\right)=\pi\left(W_{0}\right)$. In addition we have the following property $\left(Q^{*}\right)$ :

$\left(Q_{1}^{*}\right)$ for $i \in\{1,2\}$ and $v^{\prime} \in S_{\bar{W}}\left(w_{i}\right), P_{\bar{Z}}\left(\bar{\pi}\left(w_{i} v^{\prime}\right)\right)=P_{\bar{Z}}\left(a^{\prime}\right)=P_{\bar{Z}}(a)$;

$\left(Q_{2}^{*}\right)$ for any $v^{\prime} \in P_{\bar{W}}\left(w_{1}\right), \bar{\pi}\left(v^{\prime} w_{1}\right)=\bar{\pi}\left(v^{\prime} w_{2}\right)$.

Consider the sets $\mathcal{B}=\left\{b \in L_{1}(Z) \mid a b \in L_{2}(\bar{Z})\right\}$ and $\mathcal{U}=\left\{u \in L_{1}(\bar{W}) \mid \exists u^{\prime} \in\right.$ $\left.L_{1}(\bar{W}), \bar{\pi}\left(u u^{\prime}\right) \in \mathcal{B}\right\}$. It follows from properties $\left(Q_{1}^{*}\right)$ and $\left(Q_{2}^{*}\right)$ that $w_{1} \in \mathcal{U}$ if and only if $w_{2} \in \mathcal{U}$. The MS $W^{\prime}$ is defined in such a way that

$$
L_{2}\left(W^{\prime}\right) \doteq L_{2}(\bar{W}) \cup\left\{w_{1} u \mid u \in \mathcal{U}, w_{1} u \notin L_{2}(\bar{W})\right\} \cup\left\{w_{2} u \mid u \in \mathcal{U}, w_{1} u \in L_{2}(\bar{W})\right\} .
$$

The subshift $W^{\prime}$ is mixing and $L_{1}\left(W^{\prime}\right)=L_{1}(\bar{W})$. Define the map $\pi^{\prime}$ with domain $W^{\prime}$ such that $\pi^{\prime}\left(w_{i} u\right)=a$ if $w_{i} u \in L_{2}\left(W^{\prime}\right) \backslash L_{2}(\bar{W}), i \in\{1,2\}$, and $\pi^{\prime}(u v)=\bar{\pi}(u v)$ if $u v \in L_{2}(\bar{W})$. If we put $W_{0}^{\prime}=\bar{W}_{0}$, then $\pi^{\prime}\left(W_{0}^{\prime}\right)=\bar{\pi}\left(\bar{W}_{0}\right)$. Let us point out that for all $u \in \mathcal{U}$ there is a unique $w_{i}, i \in\{1,2\}$, such that $w_{i} u \in L_{2}\left(W^{\prime}\right) \backslash L_{2}(\bar{W})$. Moreover, if $w_{1}, w_{2} \in \mathcal{U}$, then $w_{i} w_{1}, w_{i} w_{2} \in L_{2}\left(W^{\prime}\right) \backslash L_{2}(\bar{W})$ for a unique $i \in\{1,2\}$, and $\pi^{\prime}\left(w_{i} w_{1}\right)=\pi^{\prime}\left(w_{i} w_{2}\right)=a$. This means that properties $\left(Q_{1}^{*}\right)$ and $\left(Q_{2}^{*}\right)$ are also satisfied with respect to $\pi^{\prime}$ and $W^{\prime}$.

Let us show that $\pi^{\prime}$ is a two-block conjugacy from $W^{\prime}$ to $Z_{a}^{*}$. First notice that for any $b \in L_{1}(Z)$ the blocks $b^{\prime} b$ in $L_{2}\left(Z_{a}^{*}\right)$ are those in $L_{2}(Z)$, and only in the case when $b \in \mathcal{B}$ is the block $a b$ added to the last set. Furthermore, since $\bar{\pi}$ is left resolving, the map $\pi^{\prime}$ produces all the blocks $b^{\prime} a \in L_{2}\left(Z_{a}^{*}\right), b^{\prime} \in P_{Z}\left(a^{\prime}\right)$, and the block $a a$ if $a^{\prime} \in \mathcal{B}$. This shows that $\pi^{\prime}\left(W^{\prime}\right) \subseteq Z_{a}^{*}$. In an analogous way as in Proposition 3.5 we can show that for every $b \in L_{1}\left(Z_{a}^{*}\right), v \in L_{1}\left(W^{\prime}\right)$, and $b^{\prime} \in P_{Z_{a}^{*}}(b)$ with $\pi^{\prime}(v \bar{v})=b$ for some $\bar{v} \in S_{W^{\prime}}(v)$, there is a unique $v^{\prime} \in P_{W^{\prime}}(v)$ such that $\pi^{\prime}\left(v^{\prime} v\right)=b^{\prime}$. This means that $\pi^{\prime}$ is a left resolving factor map from $W^{\prime}$ to $Z_{a}^{*}$.

To prove that $\pi^{\prime}$ is one-to-one, since $\bar{\pi}$ is a conjugacy, we only have to look at those points $x \in Z_{a}^{*}$ such that for infinitely many coordinates we have $x_{i}=a$. In this case, if we take $i \geq 2$ with $x_{i}=a$ and $y \in W^{\prime}$ such that $\pi^{\prime}(y)=x$, then $y_{i} \in\left\{w_{1}, w_{2}\right\}$. Since $\pi^{\prime}$ is left resolving and $\pi^{\prime}\left(v w_{1}\right)=\pi^{\prime}\left(v w_{2}\right)$ for any $v \in P_{W^{\prime}}\left(w_{1}\right)$, there is a unique $y_{i-1}$ such that $\pi^{\prime}\left(y_{i-1} w_{1}\right)=\pi^{\prime}\left(y_{i-1} w_{2}\right)=x_{i-1}$. Therefore, if we use the left resolving property recursively, we conclude that $y_{1} \ldots y_{i-1}$ is uniquely determined for all $i \geq 2$. Then $y$ is unique and $\pi^{\prime}$ is a conjugacy.

We have constructed a two-block conjugacy between the nontrivial MS $W^{\prime}$ and $Z_{a}^{*}$, and an MS $W_{0}^{\prime} \subseteq W^{\prime}$ such that $L_{1}\left(W^{\prime}\right)=L_{1}\left(W_{0}^{\prime}\right)$ and $\pi^{\prime}\left(W_{0}^{\prime}\right)=\pi\left(W_{0}\right)$. To prove that $\left(\pi^{\prime}, W_{0}^{\prime}, W^{\prime}, Z_{a}^{*}, \bar{Z}\right)$ has property $\left(P^{*}\right)$ we have to verify properties $\left(P_{1}^{*}\right)$, $\left(P_{2}^{*}\right)$ and $\left(P_{3}^{*}\right)$. Since $L_{1}(Z) \subseteq L_{1}\left(Z_{a}^{*}\right)$, property $\left(P_{3}^{*}\right)$ is straightforward. On the other hand, by construction, the MS $Z_{a}^{*}$ already has both blocks in $L_{2}(\bar{Z})$ with letters in $L_{1}(Z) \cup\{a\}$, so it satisfies property $\left(P_{1}^{*}\right)$. Finally, by using the facts that $\left(\bar{\pi}, \bar{W}_{0}, \bar{W}, Z, \bar{Z}\right)$ has property $\left(P^{*}\right)$ and property $\left(Q_{2}^{*}\right)$ with respect to $\pi^{\prime}$ and $W^{\prime}$, we can prove that $\left(P_{2}^{*}\right)$ is also true.

Corollary 3.8. Let $\left(\bar{\pi}, \bar{W}_{0}, \bar{W}, Z, \bar{Z}\right)$ be a 5-tuple with property $\left(P^{*}\right)$. Then there is a 5-tuple $\left(\pi, W_{0}, W, \bar{Z}, \bar{Z}\right)$ with property $\left(P^{*}\right)$ and $\bar{\pi}\left(\bar{W}_{0}\right)=\pi\left(W_{0}\right)$.

Proof. Put $L_{1}(\bar{Z}) \backslash L_{1}(Z)=\left\{a_{1}, \ldots, a_{s}\right\}$ and suppose that for each $i \in\{1, \ldots, s\}$ there is $c \in L_{1}(Z) \cup\left\{a_{1}, \ldots, a_{i-1}\right\}$ such that $a_{i} c \in L_{2}(\bar{Z})$. By using Proposition 3.7 we construct for each $i \in\{1, \ldots, s\}$ a 5 -tuple $\left(\pi^{(i)}, W_{0}^{(i)}, W^{(i)}, Z^{(i)}, \bar{Z}\right)$ with property 
$\left(P^{*}\right)$, where $Z^{(i)}=\left(Z^{(i-1)}\right)_{a_{i}}^{*}$, and such that $\pi^{(i)}\left(W_{0}^{(i)}\right)=\bar{\pi}\left(\bar{W}_{0}\right)$. Therefore, since $Z^{(s)}=\bar{Z}$, we conclude that $\left(\pi^{(s)}, W_{0}^{(s)}, W^{(s)}, \bar{Z}, \bar{Z}\right)$ has property $\left(P^{*}\right)$ and $\pi^{(s)}\left(W_{0}^{(s)}\right)=\bar{\pi}\left(\bar{W}_{0}\right)$.

Proof of Theorem 3.1. We have already discussed the necessity of the condition, which results from Lemmas 3.2 and 3.3.

In order to prove the sufficiency, it is enough to show it for the systems $\hat{X}_{M_{t}}$ and $\hat{X}_{0}$. A simple composition and Lemma 3.3 serves to conclude. For simplicity we will denote by $V$ the mixing MS $\hat{X}_{M_{t}}$ and by $V_{0}$ the mixing subshift of finite type $\hat{X}_{0}$. We also assume that for any $a \in L_{1}(V)$ there is $a^{\prime} \in L_{1}\left(V_{0}\right)$ such that $P_{V}(a)=P_{V}\left(a^{\prime}\right)$. Both $V_{0}$ and $V$ are nontrivial.

Let $N$ be an order of the SFT $V_{0}$. Now define for each $i \in\{1, \ldots, N\}$ the following mixing MS:

$$
V_{0}^{(i)}=\left\{\left(\begin{array}{c}
x_{0} \\
\vdots \\
x_{i-1}
\end{array}\right)\left(\begin{array}{c}
x_{1} \\
\vdots \\
x_{i}
\end{array}\right) \ldots \mid\left(x_{i}\right)_{i \in \mathbb{N}} \in V, \forall j \in \mathbb{N} x_{j} \ldots x_{j+i-1} \in L_{i}\left(V_{0}\right)\right\} .
$$

Observe that the MS $V_{0}^{(N)}$ is the $N$-block presentation of $V_{0}$; in particular, $V_{0}^{(N)}$ and $V_{0}$ are conjugate. We also set $\hat{V}_{0}^{(i)}$ equal to the projection of the first $i$ coordinates of $V_{0}^{(i+1)}$. We denote this projection by $\varphi^{(i+1)}$, so $\hat{V}_{0}^{(i)}=\varphi^{(i+1)}\left(V_{0}^{(i+1)}\right)$. Notice that $\hat{V}_{0}^{(i)} \subseteq V_{0}^{(i)}$ and $\hat{V}_{0}^{(i)}$ is a nontrivial MS conjugate to $V_{0}^{(i+1)}, \varphi^{(i+1)}$ being a one-block conjugacy. Also, $L_{1}\left(V_{0}^{(i)}\right)=L_{1}\left(\hat{V}_{0}^{(i)}\right)=L_{i}\left(V_{0}\right)$.

In the first part of the proof we will produce a 5 -tuple $\left(\pi, W_{0}, W, V_{0}^{(1)}, V\right)$ with property $\left(P^{*}\right)$ and $\pi\left(W_{0}\right)=V_{0}$. To achieve it we use a recursive process.

Fix $i=N-1$. Put $W^{(N-1)}=V_{0}^{(N-1)}$ and $W_{0}^{(N-1)}=\hat{V}_{0}^{N-1}$, and consider the identity $\pi^{(N-1)}: W^{(N-1)} \rightarrow V_{0}^{(N-1)}$. It is obvious that $\pi^{(N-1)}\left(W_{0}^{(N-1)}\right)=$ $\hat{V}_{0}^{(N-1)}$. The identity is a one-block map; thus for all $v v^{\prime}, v v^{\prime \prime} \in L_{2}\left(W^{(N-1)}\right)$, $P_{\bar{Z}}\left(\pi^{(N-1)}\left(v v^{\prime}\right)\right)=P_{\bar{Z}}\left(\pi^{(N-1)}\left(v v^{\prime \prime}\right)\right)$ for an arbitrary mixing MS $\bar{Z}$ containing $V_{0}^{(N-1)}$. We conclude that $\left(\pi^{(N-1)}, W_{0}^{(N-1)}, W^{(N-1)}, V_{0}^{(N-1)}, \bar{Z}\right)$ has property $(P)$.

Consider the map $\hat{\pi}^{(N-2)}=\varphi^{(N-1)} \circ \pi^{(N-1)}: W^{(N-1)} \rightarrow \hat{V}_{0}^{(N-2)}$. It is a oneblock conjugacy, and $\hat{\pi}^{(N-2)}\left(W_{0}^{(N-1)}\right)=\varphi^{(N-1)}\left(\hat{V}_{0}^{(N-1)}\right)$ is a mixing SFT. Therefore $\left(\hat{\pi}^{(N-2)}, W_{0}^{(N-1)}, W^{(N-1)}, \hat{V}_{0}^{(N-2)}, \bar{Z}\right)$ has property $(P)$ for any mixing MS $\bar{Z}$ containing $\hat{V}_{0}^{(N-2)}$. Since $L_{1}\left(\hat{V}_{0}^{(N-2)}\right)=L_{1}\left(V_{0}^{(N-2)}\right)$, we can apply Corollary 3.6 with $\bar{Z}=V_{0}^{(N-2)}$ in order to obtain a 5-tuple $\left(\pi^{(N-2)}, W_{0}^{(N-2)}, W^{(N-2)}, V_{0}^{(N-2)}\right.$, $V_{0}^{(N-2)}$ ) with property $(P)$. Furthermore, $\pi^{(N-2)}$ is a one-block conjugacy and

$$
\pi^{(N-2)}\left(W_{0}^{(N-2)}\right)=\hat{\pi}^{(N-2)}\left(W_{0}^{(N-1)}\right)=\left\{\left(\begin{array}{c}
x_{0} \\
\vdots \\
x_{N-3}
\end{array}\right)\left(\begin{array}{c}
x_{1} \\
\vdots \\
x_{N-2}
\end{array}\right) \ldots \mid\left(x_{i}\right)_{i \in \mathbb{N}} \in V_{0}\right\} .
$$

Since $\pi^{(N-2)}$ is one-block, we can replace the last $V_{0}^{(N-2)}$ in the 5 -tuple by any mixing MS which contains it, keeping property $(P)$. 
Let us suppose that for some $j \in\{N-2, \ldots, 2\}$ we have constructed a 5 -tuple $\left(\pi^{(j)}, W_{0}^{(j)}, W^{(j)}, V_{0}^{(j)}, \bar{Z}\right)$ with property $(P), \pi^{(j)}$ is a one-block map and

$$
\pi^{(j)}\left(W_{0}^{(j)}\right)=\left\{\left(\begin{array}{c}
x_{0} \\
\vdots \\
x_{j-1}
\end{array}\right)\left(\begin{array}{c}
x_{1} \\
\vdots \\
x_{j}
\end{array}\right) \ldots \mid\left(x_{i}\right)_{i \in \mathbb{N}} \in V_{0}\right\}
$$

(here $\bar{Z}$ is any mixing MS containing $V_{0}^{(j)}$, because $\pi^{(j)}$ is one-block).

Consider $\hat{\pi}^{(j-1)}=\varphi^{(j)} \circ \pi^{(j)}$. Then the 5-tuple $\left(\hat{\pi}^{(j-1)}, W_{0}^{(j)}, W^{(j)}, \hat{V}_{0}^{(j-1)}, \bar{Z}\right)$ has property $(P)$. Moreover, $\hat{\pi}^{(j-1)}$ is a one-block conjugacy, $L_{1}\left(\hat{V}_{0}^{(j-1)}\right)=L_{1}\left(V_{0}^{(j-1)}\right)$ and

$$
\hat{\pi}^{(j-1)}\left(W_{0}^{(j)}\right)=\left\{\left(\begin{array}{c}
x_{0} \\
\vdots \\
x_{j-2}
\end{array}\right)\left(\begin{array}{c}
x_{1} \\
\vdots \\
x_{j-1}
\end{array}\right) \ldots \mid\left(x_{i}\right)_{i \in \mathbb{N}} \in V_{0}\right\} .
$$

Thus by taking $\bar{Z}=V_{0}^{(j-1)}$ we can apply Corollary 3.6 to produce a 5 -tuple

$$
\left(\pi^{(j-1)}, W_{0}^{(j-1)}, W^{(j-1)}, V_{0}^{(j-1)}, V_{0}^{(j-1)}\right)
$$

with property $(P)$, where $\pi^{(j-1)}$ is a one-block map and

$$
\pi^{(j-1)}\left(W_{0}^{(j-1)}\right)=\hat{\pi}^{(j-1)}\left(W_{0}^{(j)}\right) .
$$

In this way we prove that there is a 5 -tuple $\left(\pi^{(1)}, W_{0}^{(1)}, W^{(1)}, V_{0}^{(1)}, V\right)$ with property $(P), \pi^{(1)}$ is a one-block conjugacy and $\pi^{(1)}\left(W_{0}^{(1)}\right)=V_{0}$. Moreover, this 5-tuple has property $\left(P^{*}\right)\left(\left(P_{3}^{*}\right)\right.$ is the hypothesis, $\left(P_{2}^{*}\right)$ because $\pi$ is one-block, and $\left(P_{1}^{*}\right)$ follows from the definition of $\left.V_{0}^{(1)}\right)$. Then we can apply Corollary 3.8 to obtain a 5 -tuple $\left(\pi, W_{0}, W, V, V\right)$ with property $\left(P^{*}\right)$ and $\pi\left(W_{0}\right)=V_{0}$; that is, we have a conjugacy $\pi: W \rightarrow V$ and a mixing MS $W_{0} \subseteq W$ with $L_{1}\left(W_{0}\right)=L_{1}(W)$ such that $\pi\left(W_{0}\right)=V_{0}$. This proves the theorem.

The following corollary has been pointed out to us by Mike Boyle. It concerns the same codification problem in the two-sided case.

Corollary 3.9. Let $X_{0} \subseteq X$ be nontrivial two-sided mixing SFT. Then there is a conjugacy $\pi: Y \rightarrow X$, where $Y$ and $Y_{0}=\pi^{-1}\left(X_{0}\right)$ are two-sided mixing $M S$ with $L_{1}\left(Y_{0}\right)=L_{1}(Y)$.

Proof. Without lost of generality let $X$ be a mixing MS defined by a totally amalgamated matrix. Because $X_{0}$ is a nontrivial SFT, it contains an infinite orbit $\left\{\sigma^{n}(x): n \in \mathbb{Z}\right\}$ such that $x$ is forwardly asymptotic to a periodic point $z$ and backwardly asymptotic to a periodic point $y$. That is $x=\ldots v v v x_{-j} \ldots x_{k} w w w \ldots$, where $v$ and $w$ are defining blocks for $y$ and $z$ respectively.

Because $X$ is a mixing SFT, for $j$ and $k$ large enough, there is a word $u_{-j} \ldots u_{k}$ such that

(i) $\bar{x}=\ldots v v v u_{-j} \ldots u_{k} w w w \ldots$ is a point in $X$, and

(ii) every symbol in $L_{1}(X)$ occurs in $u_{-j} \ldots u_{k}$.

Now we have a natural isomorphism of SFT's:

$$
\varphi^{\prime}:\{y, z\} \cup\left\{\sigma^{n}(x): n \in \mathbb{Z}\right\} \rightarrow\{y, z\} \cup\left\{\sigma^{n}(\bar{x}): n \in \mathbb{Z}\right\},
$$


such that $\varphi^{\prime}(x)=\bar{x}, \varphi^{\prime}(y)=y$ and $\varphi^{\prime}(z)=z$. Since $\varphi^{\prime}$ moves no periodic points, it follows from Lemma 1.4 in $[\mathrm{BK}]$ that $\varphi^{\prime}$ can be extended to an automorphism $\varphi: X \rightarrow X$.

Now, $\varphi$ is a conjugacy such that $L_{1}\left(\varphi\left(X_{0}\right)\right)=L_{1}(X)$. Therefore, to finish the proof we can use Theorem 3.1 to produce a conjugacy $\pi: Y \rightarrow X$, where $Y$ and $Y_{0}=\pi^{-1}\left(X_{0}\right)$ are two-sided mixing MS with $L_{1}\left(Y_{0}\right)=L_{1}(Y)$.

\section{ACKNOWLEDGMENTS}

This research was partially supported by grants from FONDECYT and the ECOS-CONICYT cooperation program. The authors are thankful for the hospitality of the Laboratoire de Mathématiques de Luminy in Marseille, where this work was finished. We thank the referee for comments which allowed us to improve the first version of this work. We are very grateful to Mike Boyle for his numerous suggestions.

\section{REFERENCES}

[BFK] M. Boyle, J. Franks, B. Kitchens, Automorphisms of one-sided subshifts of finite type, Ergodic Theory and Dynamical Systems 10, 421-449 (1990). MR 91h:58037

[BK] M. Boyle, W. Krieger, Automorphisms and subsystems of the shift, Journal für die reine und angewandte Mathematik 437, 13-28 (1993). MR 95b:54051

[LM] D. Lind, B. Marcus, An Introduction to Symbolic Dynamics and Coding, Cambridge University Press, (1995). MR 97a:58050

[Wi] R.F. Williams, Classification of subshifts of finite type, Ann. of Math. 98, 120-153 (1973); erratum Vol. 99, 380-381 (1974). MR 48:9769

Departamento de Ingeniería Matemática, Universidad de Chile, Casilla 170/3 Correo 3, Santiago, Chile

E-mail address: amaass@dim.uchile.cl

E-mail address: smartine@dim.uchile.cl 